\title{
Infections in Neurosurgical Intensive Care Patients: A 3-Year Study in a Tertiary Health Care Center, North India
}

\author{
Deepa Chaturvedi ${ }^{1}$ Pragnya P. Jena ${ }^{2} \quad$ Bansidhar Tarai ${ }^{2} \quad$ Kavita Sandhu $^{1}$ \\ ${ }^{1}$ Department of Neuroanaesthesia, Max Super Speciality Hospital, \\ New Delhi, India \\ 2Department of Microbiology, Max Super Speciality Hospital, \\ Address for correspondence Bansidhar Tarai, MD, Institute of Lab \\ Medicine, Max Super Speciality Hospital, Saket, New Delhi 110017, \\ India (e-mail: bansisss@gmail.com).
} New Delhi, India

J Neuroanaesthesiol Crit Care 2019;6:30-36

\begin{abstract}
Background In the neurosurgical patient community, infection rate depends on the severity of neurological injuries at the time of presentation, which is measured by the Glasgow coma score (GCS). In addition, associated comorbidities; exposure to invasive devices such as endotracheal tube, central venous catheters, and urinary catheters; and neuroscience-specific devices, such as ventricular/lumbar catheters, increase chances of infection. We share our experience from a dedicated neurosurgical intensive care unit (NSICU) of a super speciality tertiary care hospital in north India.

Patients and Methods This is a 3-year retrospective and observational study from January 2014 to December 2016. Total 2,608 patients were admitted to NSICU during this period; 229 patients were included whose cultures were collected after 48 hours of admission and were positive. We have analyzed patient's risk factors, length of stay (LOS), outcome, organism details, and those health care-associated infections (HAI) that fulfilled the CDC (Centers for Disease Control and Prevention) criteria.

Results Out of 2,608 patients admitted, 229 were culture positive after 48 hours of admission and 53 developed HAls (53/2,608 [2.03\%]). Male-to-female ratio was 2:1. One hundred three patients had a low GCS (5-8) and 126 had a high GCS (9-15). Average LOS in ICU was 6 days, and mortality was $17.4 \%$ (40/229). A total of 57 laboratory-confirmed positive cultures were identified in 53 patients. This included 35 from urine, 15 from blood, 2 from surgical wound, and 1 from respiratory tract.

Keywords

- infections

- neurosurgical intensive care unit

- risk factors

- lumbar and ventricular drain

- Glasgow coma score Among the HAI, the rate of ventilator-associated pneumonia (VAP) was 0.22 , central line-associated bloodstream infection (CLABSI) 3.43, catheter-associated urinary tract infection (CAUTI) 5.93, and surgical site infection (SSI) $0.9 \%$.

Conclusion Neurosurgical patients are particularly vulnerable to infection because of the formidable nature of their illness, frequency of associated trauma, and presence of invasive devices. In our study, lower rate of HAls was observed because we have a dedicated NSICU, strict infection control practices, an appropriate antimicrobial stewardship program, and early shifting of neurosurgical patients to an appropriately staffed high-dependency unit/ward.
\end{abstract}

received

November 8, 2018 accepted after revision January 24, 2019 published online March 6, 2019
DOI https://doi.org/

10.1055/s-0039-1680276 ISSN 2348-0548.
Copyright $\odot 2019$ Indian Society of Neuroanaesthesiology and Critical Care
License terms

(ㄷ) (1) $\ominus \circledast$ 


\section{Introduction}

Health care-associated infections (HAIs) are most commonly associated with invasive medical devices or surgical procedures. Millions of patients are affected by HAIs worldwide every year, leading to significant morbidity and mortality as well as financial loss for health systems. ${ }^{1}$ The HAIs burden is also significantly higher in low- and middle-income nations as compared with high-income countries, especially in patients admitted to intensive care units. Urinary tract infection is the most frequent HAI in high-income countries, whereas surgical site infection (SSI) is at the forefront in the low-income group, affecting up to one-third of operated patients. In high-income countries, approximately $30 \%$ of patients in intensive care units (ICUs) are affected by at least one $\mathrm{HAI}^{2}$

In the neurosurgical and neurocritical patient population, HAI rate depends on the severity of neurological illness at presentation and the exposure to invasive devices such as endotracheal tube (ET), central venous catheters (CVCs), and urinary catheters, in addition to neuroscience specific devices, such as ventricular or lumbar catheters. Although common causes of infection in a neurosurgical ICU (NSICU) patient are the same as those for any critically ill patient, specific considerations should be made for infections because of depressed level of consciousness and surgical opening of the cranial vault. ${ }^{3}$ Most of the time, clinical signs and symptoms of neurological patients with infectious fever and noninfectious fever markedly overlap. ${ }^{4}$

A majority of neurosurgical patients requiring ICU admission comprise those who have head injuries, subarachnoid hemorrhages, or complications of neurosurgical procedures. Only a minority will be admitted with preexisting intracranial or spinal infections, whereas majority will develop infections while they are in the ICU. ${ }^{5}$ High rate of infections are associated with long ICU stay, polytrauma, indiscriminate use of antibiotics, and lumbar puncture (LP)/Ommaya drain.

Good practices for infection prevention and control measures reduce the opportunities for resistant pathogens to spread in health care facilities. ${ }^{6}$ Additionally, timely microbiological surveillance and assessment of antimicrobial resistance are important for dealing with HAIs. There is paucity of quality data in literature about the HAI rates in NSICUs in India. We are sharing our experience from a dedicated NSICU of a tertiary care center in north India.

\section{Ethical Statement}

This is a retrospective collection of data, which did not entail any direct contact with the patient; hence, the consent was waived. This study was approved by scientific and ethics committee of our hospital.

\section{Study Design and Patient Population}

This is retrospective and observational study in NSICU. It was conducted in a 506-bedded tertiary care hospital with a 13-bedded dedicated NSICU. Data were analyzed over a span of 3 years from January 2014 to December 2016.

\section{Patients and Methods}

Annual admission of patients in our NSICU is approximately 800 to 900 . During this study period, 2,608 patients were admitted into the NSICU directly from home, road traffic accident (RTA) via triage, respiratory ward, medical ward, nephrology ward, and also transferred from other hospital. Out of 2,608 patients, culture was received in 229 patients after 48 hours of admission and was positive. Patients whose initial cultures were positive within 48 hours of ICU admission were not included. Status of the patient was assessed using the Glasgow coma score (GCS) and other medical conditions on admission. Laboratory results and specific clinical indicators were used to categorize infections as per CDC (Centers for Disease Control and Prevention) nosocomial infection surveillance definitions. ${ }^{7}$ Infection control bundles were followed and analyzed on monthly basis.

\section{Sample Collection and Processing}

Samples were collected aseptically whenever there was a suspicion of infections and as a routine protocol if patients transferred from other hospitals. Samples included urine, blood, and endotracheal secretion, in addition to cerebrospinal fluid, wound aspirate, bronchoalveolar lavage fluid, pleural fluid when indicated, and stool for Clostridium difficile polymerase chain reaction (PCR) in specific cases. Swab for methicillin-resistant Staphylococcus aureus (MRSA) was taken only if the patient came from another hospital.

All samples were transported to the clinical microbiology laboratory for immediate processing. Blood was cultured in the BacT Alert 3D system (Biomerieux, France). The other samples were processed according to established standardized protocol and current Clinical and Laboratory Standards Institute (CLSI) guideline. ${ }^{8}$

Isolates were identified to the species level and antimicrobial susceptibility testing was done using Vitek Excel (Biomerieux, France). Quality control was done using American Type Culture Collection (ATCC) strain.

\section{Statistical Analysis}

Data were analyzed using spread sheet. Statistics were analyzed based on percentage, mean, and median values or interquartile ranges (IQRs), as appropriate. $p$ Values $<0.05$ were considered statistically significant. Univariate analysis was employed to determine the contribution of clinical variables to the prediction of acquisition of infections in the ICU. Also, we calculated the odds ratios of risk factors for developing HAIs in ICU.

\section{Results}

Out of 2,608 patients, culture collected from 229 patients after 48 hours of admission was positive. Out of 229 patients, male-to-female ratio was 2:1 with an age range of 1 to 94 years, and length of stay (LOS) in the ICU ranged from 2 to 60 days (-Table 1 ). The most common diagnosis on admission was intracranial hemorrhage $62(27 \%)$, followed by space-occupying lesion (SOL) 53 (23\%), spine involvement $46(20 \%)$, stroke $23(10 \%)$, RTA 20 (8\%), and subarachnoid hemorrhage 17 (7\%) (-Fig. 1). Patients admitted with low 
32 Infections in Neurosurgical Intensive Care Patients: A 3-Year Study Chaturvedi et al.

Table 1 Baseline demographic and clinical characteristics of patients (number, \%, mean, IQR)

\begin{tabular}{|c|c|c|c|c|}
\hline Variables & $n=229$ & Percentage (\%) & Mean & Median (IQR) \\
\hline \multicolumn{5}{|l|}{ Gender } \\
\hline Female & 73 & 31.9 & & \\
\hline Male & 156 & 68.1 & & \\
\hline Age $(y)$ & & & 52 y & \\
\hline $1-14$ & 6 & 2.6 & & \\
\hline $15-60$ & 131 & 57.2 & & \\
\hline $61-90$ & 92 & 40.2 & & \\
\hline \multicolumn{5}{|l|}{ GCS } \\
\hline Low GCS (3-8) & 103 & 44.9 & & \\
\hline High GCS (9-15) & 126 & 55.1 & & \\
\hline Length of ICU stay (day) & & & 6 days & $5(8-3)$ \\
\hline $2-7$ & 173 & 75.5 & & \\
\hline $8-60$ & 56 & 24.5 & & \\
\hline \multicolumn{5}{|l|}{ Locations before ICU admissions } \\
\hline From other hospitals & 19 & 8.2 & & \\
\hline Home & 94 & 41.2 & & \\
\hline RTA & 25 & 10.9 & & \\
\hline Triage & 63 & 27.5 & & \\
\hline $\begin{array}{l}\text { Transferred from various wards } \\
\text { within the hospital }\end{array}$ & 28 & 12.2 & & \\
\hline Mortality rate & 40 & 17.4 & & \\
\hline
\end{tabular}

Abbreviations: GCS, Glasgow coma score; IQR, interquartile range; RTA, road traffic accident.

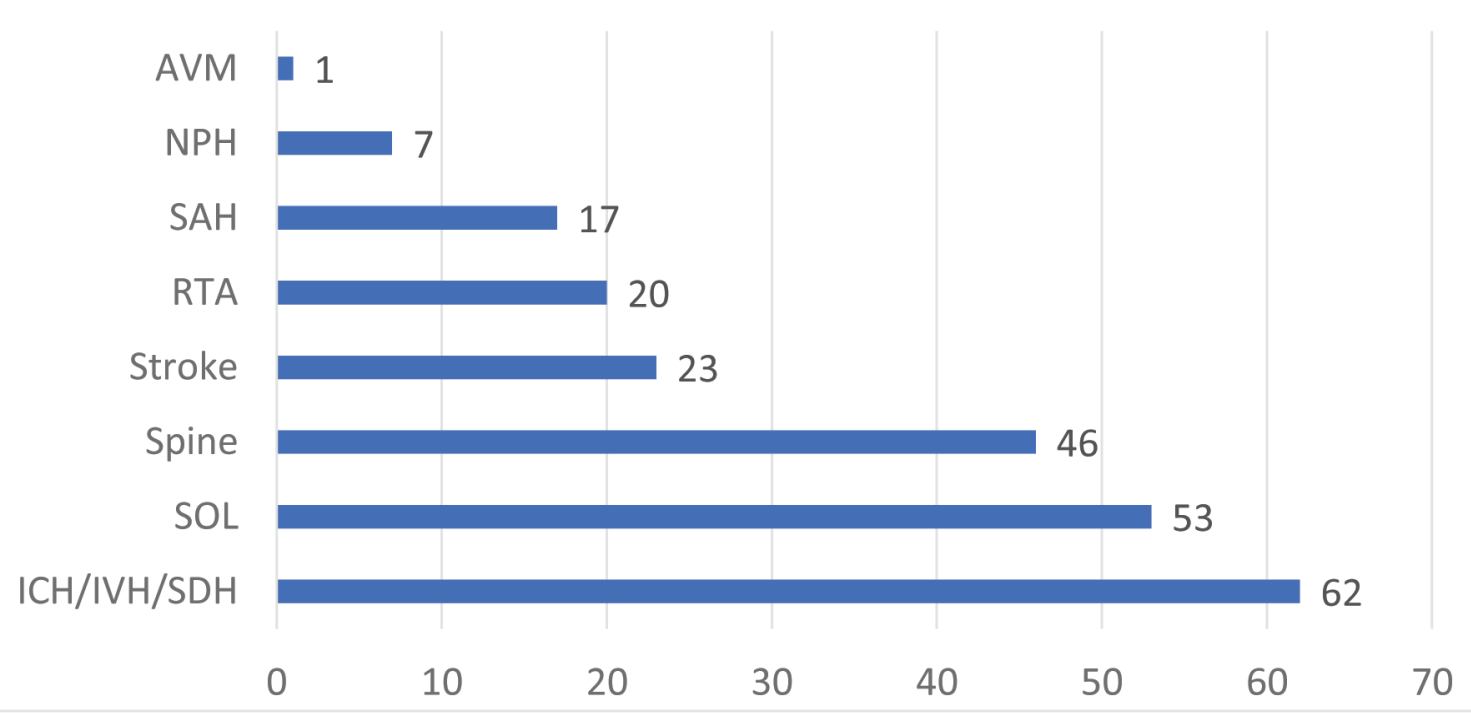

Fig. 1 Distribution of cases admitted in neurosurgical ICU $(n=229)$. AVM, arteriovenous malformation; ICH, intracerebral hemorrhage; IVH, intraventricular hemorrhage; NPH, normal-pressure hydrocephalus; RTA, road traffic accident; SAH, subarachnoid hemorrhage; SDH, subdural hemorrhage; SOL, space-occupying lesion. 
GCS were 103 (44.9\%), whereas 126 (55.1\%) patients had high GCS. For outcome analysis, patients were distributed into two subgroups according to survival status (died or transferred out to $\mathrm{HDU} / \mathrm{room} /$ ward). In the present study, mortality rate was $17.4 \%(40 / 229)$ (-Table 2).

Out of 229 patients, 167 were intubated, 115 were urinary catheterized, and 164 were on CVC in the internal jugular vein and subclavian vein. Patients transferred from other hospitals, RTAs via triage were 107 (46.6\%), patients admitted directly from home were 94 (41.2\%), and 28 (12.2\%) were transferred from various wards and ICUs within hospitals. Out of 229 culture-positive patients, 53 cases developed HAIs $(53 / 2,608$ [2.03\%]) (-Table 3). Among 53 HAIs, 29 developed early-onset (between 3 and 7 days on ICU admission), whereas 24 had late-onset infections. A total of 57 laboratoryconfirmed positive cultures were identified in 53 patients. The most common infection was urinary tract infections in 35 patients, followed by bloodstream infection (BSI) in 15 cases. One patient had two episodes of catheter-associated urinary tract infection (CAUTI) and another patient had two episodes of BSI. A total of 2 cases of skin-soft tissue infections (SSI), and only one patient had pneumonia.

\section{Microbiological Distribution of Pathogens Identified}

Thirteen different species of pathogenic microorganisms were identified out of 57 isolates ( - Fig. 2). Overall, the most common organism causing HAI was Klebsiella pneumoniae (24\%), followed by Escherichia coli (22\%). The percentage of multidrug-resistant (MDR) organism among gram-negative bacilli was $26 \%$. In infected patients, we followed culture-directed sensitivity. No case of $C$. difficile infection and MRSA was noted.

\section{Univariate Analysis of Risk Factors of HAls in the NSICU}

Using univariate analysis, statistically significant association of risk factors in development of HAIs are antibiotics use before ICU admission $(p<0.001$, odds ratio $[\mathrm{OR}]=6.775)$, presence of devices such as ET $(p<0.001, \mathrm{OR}=7.450)$, urinary catheter $(p=0.002, \mathrm{OR}=2.386)$, central line $(p<0.001$, OR $=8.022)$, location before admission $(p=0.038, \mathrm{OR}=2.063)$,

Table 2 Percentage of mortality in relation to GCS score

\begin{tabular}{|l|l|l|}
\hline GCS & $\begin{array}{l}\text { Number of deaths } \\
(n=40 / 229)\end{array}$ & Percentage (17.4\%) \\
\hline $3-8$ & 29 & 12.6 \\
\hline $9-15$ & 11 & 4.8 \\
\hline
\end{tabular}

Abbreviation: GCS, Glasgow coma score.
ICU stay $>7$ days $(p=0.003$, OR $=2.724)$, and malignancy $(p=0.001, \mathrm{OR}=8.775)(-$ Table 4$)$.

\section{Discussion}

In the present study, the incidence of infection was 2.03\%, which is similar to study by Dettenkofer et al ${ }^{9}$ but less as compared with Orsi et al. ${ }^{10}$ The study by Kumar et al showed that prevalence rate of infection in neurosurgical patients was high $(18 \%)$ when it was not a dedicated NSICU..$^{11}$ In our study, CAUTI are most prevalent followed by BSIs, whereas in other studies, the most common infection was respiratory tract, followed by infections of the urinary tract., ${ }^{9,10,12}$ We observed only a single case of ventilator-associated pneumonia (VAP) as compared with other studies. The VAP patient was operated upon in other hospital for head injury and admitted to our ICU with bilateral lower-zone consolidation. We have a low VAP rate because of the practice of closed suction and placement of subglottic ETs for all ventilated patients. Additionally, on initiation of ventilation, sterilized expiratory cassette is attached on ventilator and again changing the sterilized expiratory cassette every week for long-term ventilated patients.

Catheter-associated urinary tract infections were most common in the study by Chacon et al, ${ }^{13}$ which was similar to our study. We observed that CAUTI rate was 5.93, which was similar to a study by Orsi et al and Abulhasan et al in NSICUs. ${ }^{10,14}$ The reason for relatively higher CAUTI in our study was that neurosurgical patients required prolonged and multiple urinary catheterization. Our central line-associated bloodstream infection (CLABSI) rate was 3.43, which was much lower than that reported by Orsi et al. ${ }^{10}$ In our study, CVC lines were changed whenever there was a clinical indication, but in the study by Orsi et al, CVC line days were not mentioned. Most common organisms were gram-negative bacilli in our study, and this was similar to other studies. ${ }^{9,11,13,15,16}$ Our study shows K. pneumoniae as the most common organism in overall HAIs, whereas E. coli was most common in CAUTI. A significant finding was that no $C$. difficile infection and MRSA were observed in our study. This could be because of HAI bundles we followed for prevention of infection.

Our study showed significant association of risk factors in development of HAIs. Indiscriminate use of antibiotics in ICU, prolonged use of devices, such as ET, urinary catheter, and central venous line, and condition of patients at the time of admission, ICU stay > 7days, and malignancy played a major role in the development of infection in general ICU..$^{17,18}$ Thus patients, regardless of type of ICU, have similar rate

Table 3 Distribution of health care-associated infections (HAls) ${ }^{1,7}$

\begin{tabular}{|l|l|l|l|l|}
\hline Type of infection & No. of infections & Device days & HAI calculation & HAI rate \\
\hline VAP & 1 & 4,350 & No. of VAP/No. of ventilatory days $\times 1,000$ & 0.22 \\
\hline CLABSI & 15 & 4,361 & No. of CLABSI/No. of central line days $\times 1,000$ & 3.43 \\
\hline CAUTI & 35 & 5,895 & No. of CAUTI/No. of catheter days $\times 1,000$ & 5.93 \\
\hline SSI & 2 & 215 (No. of surgery) & No. of SSI/Total no. of surgery $\times 100$ & $0.9 \%$ \\
\hline
\end{tabular}

Abbreviations: CAUTI, catheter-associated urinary tract infection; CLABSI, central line-associated bloodstream infection; HAI, health care-associated infection; SSI, surgical site infection; VAP, ventilator-associated pneumonia. 


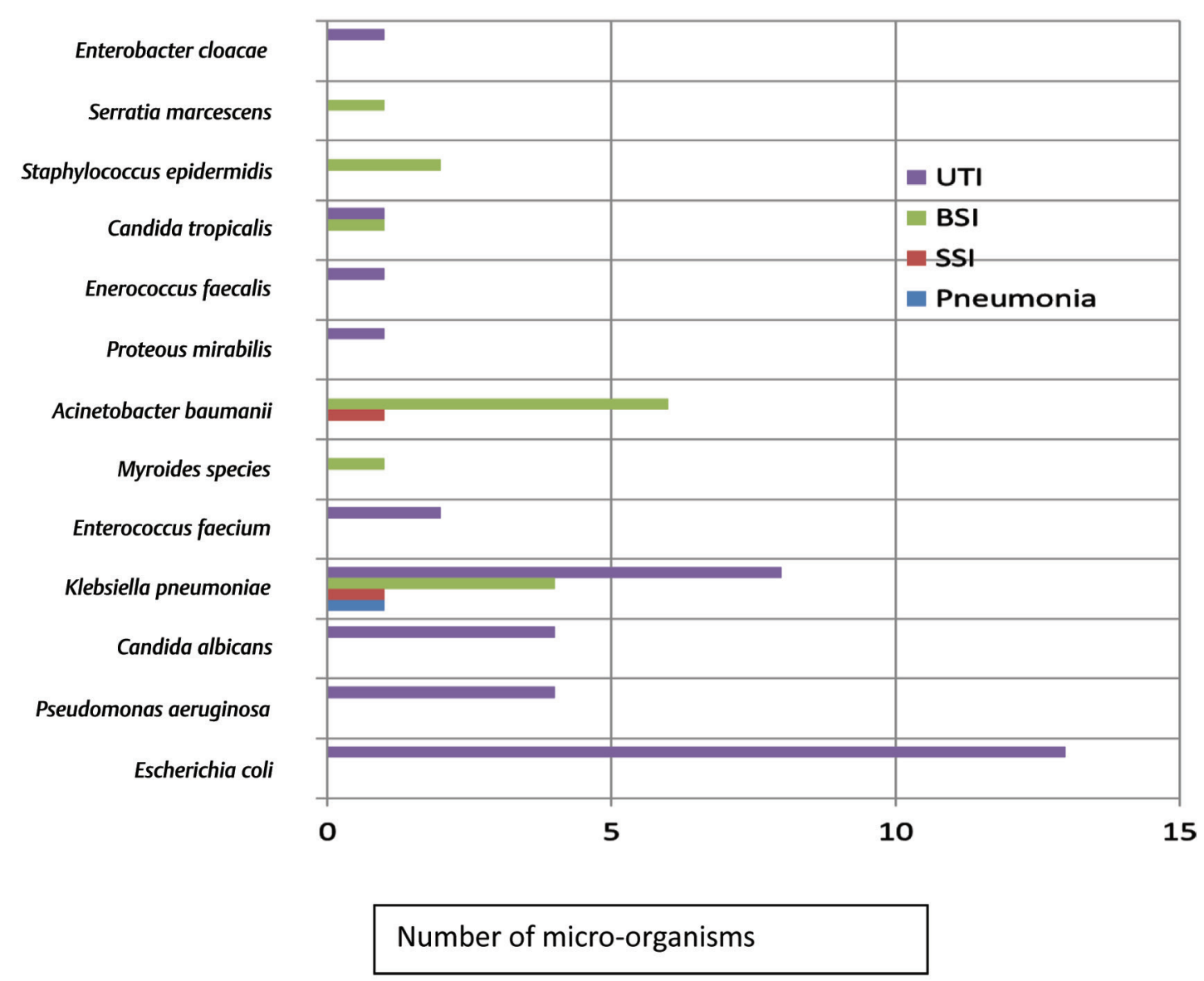

Fig. 2 Distribution of microorganisms isolated in various infections $(n=53)$. BSI, bloodstream infection; SSI, surgical site infection; UTI, urinary tract infection.

of infection with the use of invasive devices. In addition, in neuro patients, other factors, such as diabetes mellitus, hypertension, malignancy, chronic renal failure, and use of steroid for reducing peritumoral edema in space-occupying lesions pre- or postoperatively, influence the incidence of HAIs. The ICU infection may be expected to escalate because of more complex neurosurgical procedures that are prolonged, thereby exposing patients to higher incidence of infection. Because infection is related to the length of ICU stay, early shifting of neurosurgical patients to an appropriately staffed high-dependency unit/ward is likely to result in reduction in the infection rate. ${ }^{11}$

In the present study, mortality rate was $17.4 \%$, which was similar to the study by Orsi et al ${ }^{10}$ and Laborde et al. ${ }^{15}$ Deaths in low GCS cases were $12.6 \%$, whereas in high GCS these were $4.8 \%$. We observed that mortality may not directly relate to infection but may be an attributable cause of death.

The primary role of an infection-control program is to reduce the risk of hospital-acquired infection, thereby protecting patients, health care workers, and visitors. ${ }^{19}$ Antibiotic stewardship programs aim to optimize rational antibiotic use while minimizing antibiotic resistance, thus improving patient safety. This new era in HAI epidemiology is characterized by increasing scrutiny and regulation, and consumer demands for more transparency and accountability, along with calls for rapid reductions in HAI rates. ${ }^{20}$

\section{NSICU Protocol and Infection Control Practices Followed in Our Hospital}

We give prophylactic surgical antibiotic within 60 minutes before incision: (1) injectable cefuroxime single dose in clean cases, (2) cefuroxime (24 hours) and single dose of amikacin intraoperatively in spinal surgery with implant, (3) cefuroxime with amikacin and metronidazole for 72 hours in clean cerebrospinal fluid (CSF) leak cases. Infection control bundles followed in our hospital are CDC guided. There is a provision of a separate hand rub, artificial manual breathing unit (AMBU) bag, thermometer, bed pan, deep vein thrombosis (DVT) pump, and stethoscope for each bed. We follow regular communication of intensivist and clinical microbiologist on daily basis. We have separate nurses for wound care, bedsore, and SSIs, restricted visitors/visiting hours in ICU, etc. Key concerns are proper hand hygiene, pre-surgery body wash with chlorhexidine below the neck, rational antibiotic policy, optimal device site care, maximum barrier precaution, daily review, and care. 
Table 4 Univariate analysis of risk factors for neurosurgical intensive care unit-acquired infections $(n=229)$

\begin{tabular}{|c|c|c|c|c|}
\hline Risk factors & HAI (53 patients) & Non-HAI (176 patients) & $p$-Value & Odds ratio \\
\hline \multicolumn{5}{|l|}{ Use of antibiotic before NSICU admission } \\
\hline Yes & $41(18 \%)$ & $59(25 \%)$ & \multirow[t]{2}{*}{$<0.001$} & \multirow[t]{2}{*}{6.775} \\
\hline No & $12(5.2 \%)$ & $117(51 \%)$ & & \\
\hline \multicolumn{5}{|l|}{ Endotracheal intubation } \\
\hline Yes & $53(23 \%)$ & $114(50 \%)$ & \multirow[t]{2}{*}{$<0.001$} & \multirow[t]{2}{*}{7.450} \\
\hline No & 0 & $62(27 \%)$ & & \\
\hline \multicolumn{5}{|l|}{ Urinary catheterization } \\
\hline Yes & $53(23 \%)$ & $62(27 \%)$ & \multirow[t]{2}{*}{0.002} & \multirow[t]{2}{*}{2.386} \\
\hline No & 0 & $114(50 \%)$ & & \\
\hline \multicolumn{5}{|l|}{ Presence of central line } \\
\hline Yes & $53(23 \%)$ & $111(48 \%)$ & \multirow[t]{2}{*}{$<0.001$} & \multirow[t]{2}{*}{8.022} \\
\hline No & 0 & $65(28 \%)$ & & \\
\hline \multicolumn{5}{|l|}{ Location before admission } \\
\hline $\begin{array}{l}\text { Within the hospital wards/other hospital/ } \\
\text { RTA/emergency }\end{array}$ & $38(16 \%)$ & $97(42 \%)$ & \multirow[t]{2}{*}{0.038} & \multirow[t]{2}{*}{2.063} \\
\hline Home & $15(6 \%)$ & $79(34 \%)$ & & \\
\hline \multicolumn{5}{|l|}{ Age $(y)$} \\
\hline$<60$ & $32(14 \%)$ & $105(45.8 \%)$ & \multirow[t]{2}{*}{1.000} & \multirow[t]{2}{*}{1.030} \\
\hline$>61$ & $21(9 \%)$ & $71(31 \%)$ & & \\
\hline \multicolumn{5}{|l|}{ Sex } \\
\hline Male & $35(15 \%)$ & $121(52.8 \%)$ & \multirow[t]{2}{*}{0.738} & \multirow[t]{2}{*}{0.883} \\
\hline Female & $18(7.8 \%)$ & 55 (24\%) & & \\
\hline \multicolumn{5}{|l|}{ Malignancy } \\
\hline Yes & $7(3 \%)$ & $3(1 \%)$ & \multirow[t]{2}{*}{0.001} & \multirow[t]{2}{*}{8.775} \\
\hline No & $46(20 \%)$ & $173(75 \%)$ & & \\
\hline \multicolumn{5}{|l|}{ Length of ICU stay } \\
\hline$\geq 7$ & $24(10 \%)$ & $41(18 \%)$ & \multirow[t]{2}{*}{0.003} & \multirow[t]{2}{*}{2.724} \\
\hline$<7$ & 29 (12.6\%) & $135(60 \%)$ & & \\
\hline \multicolumn{5}{|l|}{ GCS } \\
\hline Low GCS (3-8) & $17(7 \%)$ & $83(36 \%)$ & \multirow[t]{2}{*}{0.080} & \multirow[t]{2}{*}{0.595} \\
\hline High GCS (9-15) & $33(14 \%)$ & $96(42 \%)$ & & \\
\hline
\end{tabular}

Abbreviations: GCS, Glasgow coma score; HAI, health care-associated infection; ICU, intensive care unit; NSICU, neurosurgical intensive care unit; RTA, road traffic accident.

\section{Conflict of Interest}

None declared.

\section{References}

1 Healthcare-associated infections. Available at: https://www. cdc.gov/hai/surveillance/index.html. Accessed April 5, 2018

2 World Health Organization. Health care-associated infections fact sheet. Available at: int/gpsc/countrywork/gpsc_ccisc_ fact_sheet_en.pdf. 2016. Accessed April 7, 2018.

3 Laws C, Jallo J. Fever and infection in the neurosurgical intensive care unit. JHN Journal 2010;5(2); article 5

4 Laifer G, Wasner M, Sendi P, et al. Dynamics of serum procalcitonin in patients after major neurosurgery. Clin Microbiol Infect 2005;11(8):679-681
5 Brown EM. Neurosurgical infections in intensive care unit patients. In: Rello J, Kollef M, Díaz E, Rodríguez A, eds. Infectious Diseases in Critical Care. Berlin, Heidelberg, Germany: Springer; 2007:581-594

6 The Review on Antimicrobial Resistance. Infection prevention, control and surveillance: limiting the development and spread of drug resistance, 2016. Available at: https://amrreview.org/sites/default/files/Health\%20infrastructure\%20 and\%20surveillance\%20final\%20version_LR_NO\%20CROPS. pdf. Accessed April 5, 2018

7 CDC/NHSN Surveillance Definitions for Specific Types of Infections. Available at: https://www.cdc.gov/nhsn/pdfs/pscmanual/17pscnosinfdef_current.pdf

8 Clinical and Laboratory Standard Institute, Performance Standards for Antimicrobial Susceptibility Testing. 28th ed. January 2018; M100 
9 Dettenkofer M, Ebner W, Hans FJ, et al. Nosocomial infections in a neurosurgery intensive care unit. Acta Neurochir (Wien) 1999;141(12):1303-1308

10 Orsi GB, Scorzolini L, Franchi C, Mondillo V, Rosa G, Venditti M. Hospital-acquired infection surveillance in a neurosurgical intensive care unit. J Hosp Infect 2006;64(1):23-29

11 Kumar S, Sen P, Gaind R, et al. Prospective surveillance of device-associated healthcare-associated infection in an intensive care unit of a tertiary care hospital in New Delhi, India. Am J Infect Control 2018;46(2):202-206

12 O'Shea M, Crandon I, Harding H, Donaldson G, Bruce C, Ehikhametalor K. Infections in neurosurgical patients admitted to the intensive care unit at the University Hospital of the West Indies. West Indian Med J 2004;53(3):159-163

13 Chacon J, Bigbee J, Fisher V, Ostrosky-Zeichner L. https:// www.researchgate.net/publication/268123763_HealthcareAssociated_Infections_HAIs_in_a_Neurosurgical_Intensive_ Care_Unit_NSICU. Accessed March 1, 2018

14 Abulhasan YB, Rachel SP, Châtillon-Angle MO, et al. Healthcare-associated infections in the neurological intensive care unit: results of a 6 -year surveillance study at a major tertiary care center. Am J Infect Control 2018;46(6):656-662
15 Laborde G, Grosskopf U, Schmieder K, et al. Nosocomial infections in a neurosurgical intensive care unit [in German]. Anaesthesist 1993;42(10):724-731

16 Tarai B, Jain D, Das P, Budhiraja S. Paired blood cultures increase the sensitivity for detecting pathogens in both inpatients and outpatients. Eur J Clin Microbiol Infect Dis 2018;37(3):435-441

17 Iwuafor AA, Ogunsola FT, Oladele RO, et al. Incidence, clinical outcome and risk factors of intensive care unit infections in the Lagos University Teaching Hospital (LUTH), Lagos, Nigeria. PLoS One 2016;11(10):e0165242

18 Phu VD, Wertheim HFL, Larsson M, et al. Burden of hospital acquired infections and antimicrobial use in Vietnamese adult intensive care units. PLoS One 2016;11(1):e0147544

19 Douglas M. Bennett's Principles and Practice of Infectious Diseases. 7th ed. Philadelphia, PA: Churchill Livingstone Elsevier; 1600:3669

20 Edmond M, Eickhoff TC. Who is steering the ship? External influences on infection control programs. Clin Infect Dis 2008;46(11):1746-1750 